\title{
Structure, stability, depolarized light scattering, and vibrational spectra of fullerenols from all-electron density-functional-theory calculations
}

\author{
Roberto Rivelino, ${ }^{1, *}$ Thaciana Malaspina, ${ }^{2}$ and Eudes E. Fileti ${ }^{3, \dagger}$ \\ ${ }^{1}$ Instituto de Física, Universidade Federal da Bahia, 40210-340 Salvador, Bahia, Brazil \\ ${ }^{2}$ Instituto de Química, Universidade de São Paulo 05513-970 São Paulo, São Paulo, Brazil \\ ${ }^{3}$ Centro de Ciências Naturais e Humanas, Universidade Federal do ABC, 09210-270 Santo André, São Paulo, Brazil
}

(Received 4 July 2008; published 13 January 2009)

\begin{abstract}
We have investigated the stability, electronic properties, Rayleigh (elastic), and Raman (inelastic) depolarization ratios, infrared and Raman absorption vibrational spectra of fullerenols $\left[\mathrm{C}_{60}(\mathrm{OH})_{n}\right]$ with different degrees of hydroxylation by using all-electron density-functional-theory (DFT) methods. Stable arrangements of these molecules were found by means of full geometry optimizations using Becke's three-parameter exchange functional with the Lee, Yang, and Parr correlation functional. This DFT level has been combined with the 6-31G $(d, p)$ Gaussian-type basis set, as a compromise between accuracy and capability to treat highly hydroxylated fullerenes, e.g., $\mathrm{C}_{60}(\mathrm{OH})_{36}$. Thus, the molecular properties of fullerenols were systematically analyzed for structures with $n=1,2,3,4,8,10,16,18,24,32$, and 36. From the electronic structure analysis of these molecules, we have evidenced an important effect related to the weak chemical reactivity of a possible $\mathrm{C}_{60}(\mathrm{OH})_{24}$ isomer. To investigate Raman scattering and the vibrational spectra of the different fullerenols, frequency calculations are carried out within the harmonic approximation. In this case a systematic study is only performed for $n=1-4,8,10,16,18$, and 24 . Our results give good agreements with the expected changes in the spectral absorptions due to the hydroxylation of fullerenes.
\end{abstract}

DOI: 10.1103/PhysRevA.79.013201

PACS number(s): 61.48.-c, 31.10.+z, 33.20.Fb, 33.20.Tp

\section{INTRODUCTION}

Hydroxylated fullerenes, so-called fullerenols or fullerols, have emerged as a class of exciting fullerene-doped compounds with important applications in tissue culture and in vivo [1-4], as well as in molecular optical devices [5-8]. Regarding biomedical technologies, the phototoxicity of the fullerenols has been recognized as an essential feature for cancer therapeutics [9-12]. In the past few years, several biological activities and methods to synthesize fullerenols $[13,14]$ have been reported. Also, recent optical spectroscopy studies $[8,15]$ of these molecules have evidenced the possibility of tuning their absorption properties by controlling the number of hydroxyl groups attached to the external fullerene surface. On the other hand, experimentally, it is a very difficult task to determine the exact molecular structure of the various stoichiometric compositions of the fullerenols $\left[\mathrm{C}_{60}(\mathrm{OH})_{n}\right]$, which can be stabilized as a function of the hydroxylation [13-16].

From the theoretical framework, the great challenge is finding the global minima of the diverse phases of the isolated $\mathrm{C}_{60}(\mathrm{OH})_{n}$ molecules. These systems present a special structural flexibility due to the rotation of the $\mathrm{OH}$ groups around the $\mathrm{C}-\mathrm{O}$ bonds and the distribution of these groups onto different carbon sites of the fullerene surface. For this reason, the localization of all stationary points on their potential energy surfaces is beyond the capability of $a b$ initio geometry optimizations [17]. Also, it is impossible to rely on chemical experience or intuition alone. Early initiatives to investigate the structure and stability of fullerenols in con-

\footnotetext{
*rivelino@ufba.br

†fileti@ufabc.edu.br; FAX: +55 7132836606
}

nection with the electronic structures were carried out using semiempirical methods $[18,19]$. Currently, these methods have been employed to determine stable geometrical configurations of fullerenols and, in combination with densityfunctional-theory (DFT) approaches, to describe their electronic properties [20,21]. Despite these computational efforts, our understanding of the structural and electronic properties of fullerenols is much less complete. Indeed, the reactivity of highly polyhydroxylated fullerenes seems to be a controversial issue by considering the mechanisms behind the production of reactive oxygen species [9-12]. Hence, on account of the importance and increasing interest in fullerene-based nanomaterials, we have performed a systematic investigation of many different properties of representative stable species of fullerenols obtained from all-electron DFT calculations.

The present work will focus on fundamental properties, such as the static electric polarizability that is connected with important molecular features [22], Rayleigh and Raman depolarized light-scattering ratios that provide information on the size and structure [23,24], and infrared (ir) and Raman vibrational spectra, which are of utmost importance to characterize a variety of stable structures of fullerenols $[14,16,25]$. Our calculations have been carried out using allelectron DFT with one of the most popular hybrid functionals, i.e., Becke's three-parameter exchange functional with the Lee, Yang, and Parr correlation functional (B3LYP) $[26,27]$. This DFT level has been combined with the $6-31 \mathrm{G}(d, p)$ Gaussian-type basis set as a compromise between accuracy and capability to treat hundreds of atoms, as in the case of the $\mathrm{C}_{60}(\mathrm{OH})_{36}$ system. Previously, we have noticed the B3LYP/6-31G $(d, p)$ method gives a good performance in determining optimized geometries of fullerenols containing different degrees of hydroxylation in comparison 
with norm-conserving pseudopotential DFT schemes [15]. Thus, the structural stability is analyzed here for $\mathrm{C}_{60}(\mathrm{OH})_{n}$ structures with $n=1,2,3,4,8,10,16,18,24,32$, and 36 by performing full geometry optimizations at the B3LYP/6-31G $(d, p)$ level.

This paper is organized as follows: In Sec. II we give detailed information on the computational strategy and methods employed for the calculations. In Sec. III we present the results analyzing properties in connection with the structural features and stability of the fullerenols. Also, a brief discussion will be made on the electronic states of these compounds. Both the ir and Raman spectra are systematically investigated as a function of the hydroxyl group number. Finally, in Sec. IV the summary and conclusions are presented.

\section{COMPUTATIONAL DETAILS AND METHODS}

As discussed earlier, it is not feasible to scan the electronic potential energy surfaces of the diverse configurations of the isolated $\mathrm{C}_{60}(\mathrm{OH})_{n}$ molecules [17] in order to find the global minima. Thus, we will consider representative lower energy configurations with $n=1-4,8,10,16,18,24,32$, and 36. The stationary points are found by performing allelectron DFT calculations with the hybrid functional B3LYP $[26,27]$, which is widely known to give good performance in many applications involving fullerene-based systems $[28,29]$. We employ the Gaussian-type basis set 6-31G $(d, p)$ that includes polarization functions [e.g., 900 contracted Gaussian functions (CGFs) for pure $\mathrm{C}_{60}$ ], to obtain a fairly accurate description of these systems. Notice that our limit is extended up to $1380 \mathrm{CGFs}$ for $\mathrm{C}_{60}(\mathrm{OH})_{24}$, along with frequency calculations, and $1620 \mathrm{CGFs}$ for the gradient calculations of our largest structure, $\mathrm{C}_{60}(\mathrm{OH})_{36}$. Frequency calculations are carried out within the harmonic approximation to obtain the vibrational spectra with ir and Raman absorptions, as well as zero-point vibration energy (ZPVE) corrections. Our considered structures present real frequencies in the range of $80-3800 \mathrm{~cm}^{-1}$. We employ methods and algorithms as implemented in the GAUSSIAN03 package [30]. This is our best way to compromise on the quality of the present calculations.

The mean dipole polarizability $(\bar{\alpha})$, anisotropy $(\Delta \alpha)$, and their derivatives with respect to the normal coordinates $\left(\bar{\alpha}^{\prime}\right.$ and $\Delta \alpha^{\prime}$ ) were calculated in order to make predictions of Rayleigh and Raman light-scattering properties of these complexes. The static polarizability and first hyperpolarizability tensors were calculated within the finite-field approximation [31-33] in which the total energy of the system is calculated both in the absence and in the presence of a weak electric field. Thus, the electric properties are determined as derivatives of the energy with respect to the electric field. The components of the strengths used here are 0.002 a.u.. The differential cross section for depolarized Rayleigh scattering observed at right angles to the incident linearly polarized [34] beam is completely determined by the activities $[23,35,36]$

$$
R_{p \perp}=45(\bar{\alpha})^{2}+7(\Delta \alpha)^{2} \quad \text { and } R_{p \|}=6(\Delta \alpha)^{2},
$$

with $\bar{\alpha}$ and $\Delta \alpha$ defined as

$$
\bar{\alpha}=\frac{1}{3}\left(\alpha_{x x}+\alpha_{y y}+\alpha_{z z}\right)
$$

and

$$
\begin{aligned}
(\Delta \alpha)^{2}= & \frac{1}{2}\left[\left(\alpha_{x x}-\alpha_{y y}\right)^{2}+\left(\alpha_{y y}-\alpha_{z z}\right)^{2}+\left(\alpha_{z z}-\alpha_{x x}\right)^{2}\right] \\
& +3\left[\left(\alpha_{x y}\right)^{2}+\left(\alpha_{x z}\right)^{2}+\left(\alpha_{y z}\right)^{2}\right],
\end{aligned}
$$

where $\alpha_{i j}$ are the components of the polarizability tensor $\boldsymbol{\alpha}$ along the molecular axis of the system.

The quantities most frequently observed experimentally are the depolarization ratios (or degree of depolarization) for both natural and plane-polarized light that are, respectively, given by

$$
\begin{gathered}
\sigma_{n}=\frac{6(\Delta \alpha)^{2}}{45(\bar{\alpha})^{2}+7(\Delta \alpha)^{2}}, \\
\sigma_{p}=\frac{3(\Delta \alpha)^{2}}{45(\bar{\alpha})^{2}+4(\Delta \alpha)^{2}} .
\end{gathered}
$$

The largest value of the degree of depolarization occurs for the most anisotropic case. As $\bar{\alpha}$ cannot vanish, for Rayleigh scattering the maxima depolarization values are $\sigma_{n}^{\max }=\frac{1}{2}$ and $\sigma_{p}^{\max }=\frac{1}{3}$. For completeness, it is interesting to obtain the depolarization for circularly polarized light, which is given by $\sigma_{c}=\sigma_{n} / 1-\sigma_{n}$, with maximum depolarization value $\sigma_{c}^{\max }=1$. Raman scattering properties are obtained by using a similar formalism, but replacing $\bar{\alpha}$ and $\Delta \alpha$ by the derivatives $\bar{\alpha}^{\prime}$ and $\Delta \alpha^{\prime}[35,36]$.

\section{RESULTS AND DISCUSSION}

\section{A. Structure, stability, and reactivity of fullerenols}

The fully optimized structures of the fullerenols, $\mathrm{C}_{60}(\mathrm{OH})_{n}$, with $n=1-4,8,10,16,18,24,32$, and 36 are illustrated in Fig. 1. The stability of these hydroxylated fullerenes are analyzed from their calculated complexation energies, at the B3LYP/6-31G $(d, p)$ level of DFT, which are given by

$$
\Delta E=E\left[\mathrm{C}_{60}(\mathrm{OH})_{n}\right]-E\left[\mathrm{C}_{60}\right]-n E_{\mathrm{OH}},
$$

where $E\left[\mathrm{C}_{60}(\mathrm{OH})_{n}\right]$ is the total energy of the hydroxylated fullerene, $E\left[\mathrm{C}_{60}\right]$ is the total energy of the bare fullerene, and $E_{\mathrm{OH}}$ is the total energy of an isolated hydroxyl group. The values of $\Delta E, \Delta E / n$, and the corresponding ZPVE corrections are displayed in Table I. As expected for these systems, the complexation energies present more negative values with the increase of the number of hydroxyl groups attached to the fullerene surface. Indeed, as can be noticed in Table I, the magnitude of these energy values increases dramatically for higher hydroxylated fullerenes. On the other hand, a large number of hydroxyl groups $(n>36)$ adsorbed on the fullerene surface may lead to unstable open-cage structures [16].

To better understand the stability of these complexes, it is interesting to analyze the complexation energy per hydroxyl 

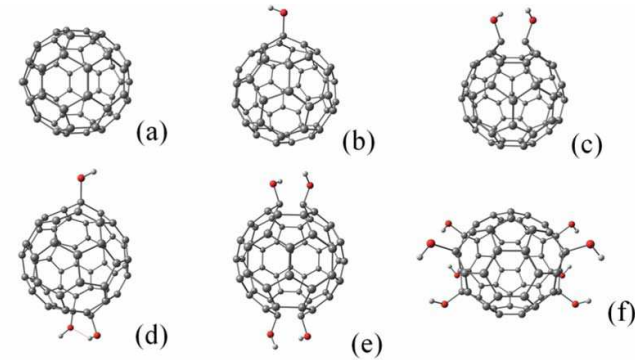

(e)
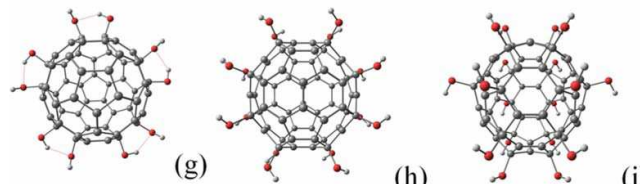

(h)
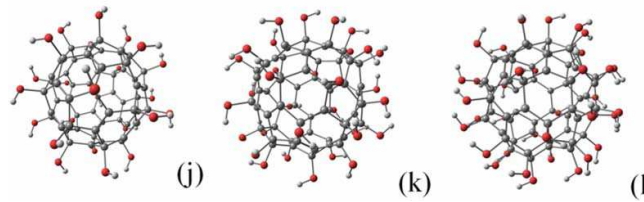

(1)

FIG. 1. (Color online) Optimized structures of (a) $\mathrm{C}_{60}$ and different stable fullerenols at the B3LYP/6-31G $(d, p)$ level of theory. (b) $\mathrm{C}_{60} \mathrm{OH}$, (c) $\mathrm{C}_{60}(\mathrm{OH})_{2}$, (d) $\mathrm{C}_{60}(\mathrm{OH})_{3}$, (e) $\mathrm{C}_{60}(\mathrm{OH})_{4}$, (f) $\mathrm{C}_{60}(\mathrm{OH})_{8}, \quad(\mathrm{~g}) \mathrm{C}_{60}(\mathrm{OH})_{10}, \quad$ (h) $\mathrm{C}_{60}(\mathrm{OH})_{16}, \quad$ (i) $\mathrm{C}_{60}(\mathrm{OH})_{18}, \quad$ (j) $\mathrm{C}_{60}(\mathrm{OH})_{24},(\mathrm{k}) \mathrm{C}_{60}(\mathrm{OH})_{32},(\mathrm{l}) \mathrm{C}_{60}(\mathrm{OH})_{36}$.

group $(\Delta E / n)$, from which we can define the adsorption energy as $-\Delta E / n$. For example, for $n=2-36$, the average value is $2.3 \pm 0.2 \mathrm{eV}$, indicating a similar stability of these cages. It is more interesting to realize in Table I that the adsorption energy of low to highly hydroxylated fullerenes weakly depends on the number of hydroxyl groups attached to the fullerene surface. Comparing the adsorption energy of $\mathrm{C}_{60}(\mathrm{OH})_{2}(2.43 \mathrm{eV})$ with the value calculated for $\mathrm{C}_{60}(\mathrm{OH})_{3}$

TABLE I. Total energies (in a.u.), complexation energies (in $\mathrm{eV}$ ), and zero-point vibration energy (ZPVE) corrections (in eV) of $\mathrm{C}_{60}(\mathrm{OH})_{n}$ systems calculated with B3LYP/6-31G $(d, p)$.

\begin{tabular}{lcccc}
\hline \hline$n$ & Total energy $^{\mathrm{a}}$ & $\Delta E$ & $\Delta E / n$ & ZPVE $^{\mathrm{b}}$ \\
\hline 0 & -2286.174031 & 0.00 & & 0.02 \\
1 & -2361.960942 & -1.65 & -1.65 & 0.02 \\
2 & -2437.626616 & -4.87 & -2.43 & 0.02 \\
3 & -2513.352908 & -6.45 & -2.15 & 0.02 \\
4 & -2589.079201 & -9.68 & -2.41 & 0.02 \\
8 & -2892.599362 & -16.76 & -2.09 & 0.02 \\
10 & -3044.344291 & -24.73 & -2.47 & 0.02 \\
16 & -3499.160368 & -37.23 & -2.32 & 0.03 \\
18 & -3650.667094 & -38.70 & -2.15 & 0.03 \\
24 & -4105.699241 & -57.09 & -2.37 & 0.03 \\
32 & -4711.947160 & -68.89 & -2.15 & \\
36 & -5015.179538 & -77.79 & -2.16 & \\
\hline
\end{tabular}

${ }^{\mathrm{a}}$ The total energy of one $\mathrm{OH}$ is -75.7262924 a.u. with $\mathrm{B} 3 \mathrm{LYP} / 6-31 \mathrm{G}(d, p)$.

${ }^{\mathrm{b}}$ Frequency calculations for $\mathrm{C}_{60}(\mathrm{OH})_{32,36}$ were not performed at this level of theory.

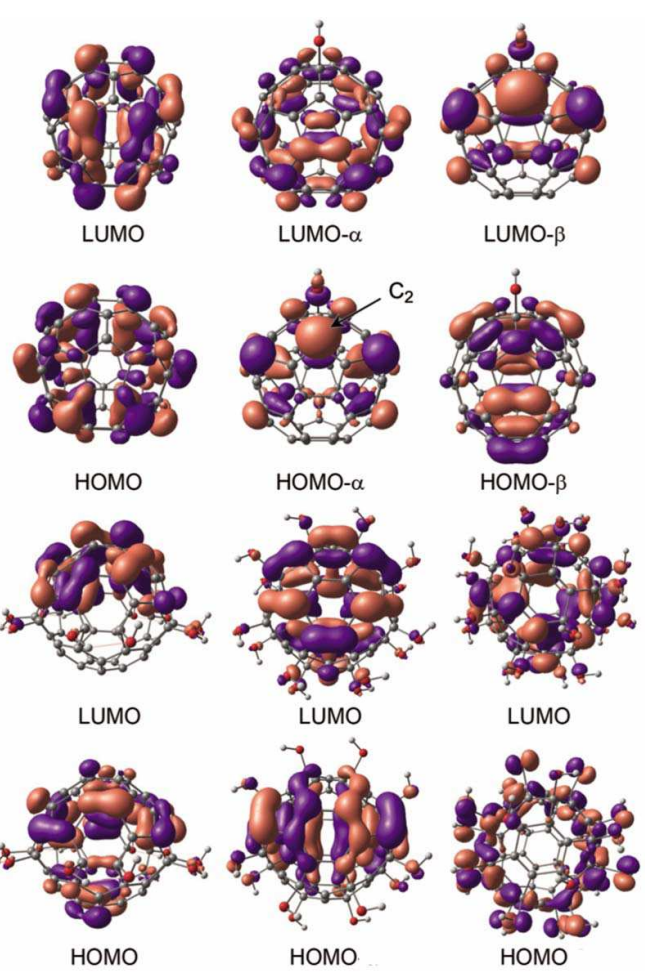

FIG. 2. (Color online) Illustration of the highest occupied molecular orbital (HOMO) and lowest unoccupied molecular orbital (LUMO) of some fullerenols species. (Left, top) $\mathrm{C}_{60}$; (middle, top) $\alpha$-spin orbital; and (right, top) $\beta$-spin orbital of $\mathrm{C}_{60} \mathrm{OH}$. (Left, bottom) $\mathrm{C}_{60}(\mathrm{OH})_{10}$; (middle, bottom) $\mathrm{C}_{60}(\mathrm{OH})_{16}$; and (right, bottom) $\mathrm{C}_{60}(\mathrm{OH})_{24}$. All these orbitals have been obtained for the same isosurface. The label $\mathrm{C}_{2}$ indicates the first neighbor to the $\mathrm{OH}$ group of $\mathrm{C}_{60} \mathrm{OH}$, belonging to a pentagon vertex of the fullerene cage (see details in Fig. 3).

(2.15 eV), we observe a small reduction of ca. $0.3 \mathrm{eV}$. Going to $\mathrm{C}_{60}(\mathrm{OH})_{4}$ this energy gives practically the same value obtained for $\mathrm{C}_{60}(\mathrm{OH})_{2}$. After this point, the adsorption energy turns to decrease to $2.09 \mathrm{eV}$ in $\mathrm{C}_{60}(\mathrm{OH})_{8}$, and increase again to $2.47 \mathrm{eV}$ in $\mathrm{C}_{60}(\mathrm{OH})_{10}$. Of course, this analysis will also depend on the specific distribution of hydroxyl groups on the carbon sites. However, as we have previously investigated [15], the relative stability varies around $10 \%$ for different stable isomers of fullerenols. Note that for these complexes the ZPVE corrections contribute to a very mild value, between 0.02 and $0.03 \mathrm{eV}$, which may indicate a high structural stability of these carbon cages. In the case of polyhydroxylated fullerenes containing numerous hydroxyl groups (e.g., $n=32,36)$ there will be much less stable isomers although some of these species have a similar stability in comparison with the moderately hydroxylated systems (see Ref. [21]).

Now we will analyze the stability of the fullerenols in connection with their electron structure within the oneparticle framework. In Fig. 2 we illustrate both the highest occupied and lowest unoccupied molecular orbitals (HOMO and LUMO, respectively) of some fullerenols species. As depicted in Fig. 2, the open-shell $\mathrm{C}_{60} \mathrm{OH}$ molecule exhibits a localized occupied electron state, namely, the HOMO- $\alpha$, in 


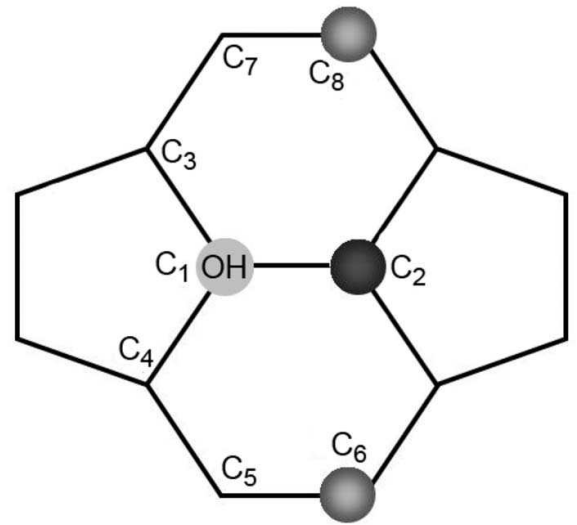

FIG. 3. Sketch of the $\mathrm{C}_{60} \mathrm{OH}$ molecule top. The spin density at the first-neighbor site $\left(\mathrm{C}_{2}\right)$ to the $\mathrm{OH}$-adsorbed site $\left(\mathrm{C}_{1}\right)$, belonging to a pentagon vertex of the fullerene cage, is higher than the other first-neighbor sites $\left(\mathrm{C}_{3}\right.$ and $\left.\mathrm{C}_{4}\right)$, belonging to the hexagon rings vertices. Interestingly, these sites give isotropic $\mathrm{FC}$ coupling constants of $4.44 \mathrm{G}$, whereas for $\mathrm{C}_{6}$ and $\mathrm{C}_{8} \mathrm{FC}=11.66 \mathrm{G}$.

the adjacent carbon atom (labeled as $\mathrm{C}_{2}$ in Fig. 2) to the $\mathrm{OH}$-adsorbed site. This first-neighbor carbon site belongs to a pentagon vertex of the fullerene cage, as sketched in Fig. 3, and its electron spin density is related to the HOMO- $\alpha$ and LUMO- $\beta$. Correspondingly, the spin density in this site is directly related to the highest isotropic Fermi contact (FC) coupling (calculated as being 23.58 Gauss) in $\mathrm{C}_{60} \mathrm{OH}$. On the other hand, the other two first-neighbor carbon atoms to the $\mathrm{OH}$-adsorbed site belonging to the hexagon vertices give mild FC coupling constants of $4.44 \mathrm{G}$. This type of electron state of $p$ character can be responsible for the high capacity of polyhydroxylation observed for these molecules [15]. This reason has led us to investigate the $\mathrm{C}_{60}(\mathrm{OH})_{2}, \mathrm{C}_{60}(\mathrm{OH})_{4}$, and $\mathrm{C}_{60}(\mathrm{OH})_{10}$ isomers containing two adjacent hydroxyl groups, as displayed in Fig 1.

The single-electron analysis can also evidence an important feature related to the reactivity of higher hydroxylated fullerenes. For instance, in our proposed isomer of $\mathrm{C}_{60}(\mathrm{OH})_{10}$, while the HOMO states are well distributed in the fullerene surface, the LUMO states are well localized only in one side of the carbon cage. This may indicate a preference for chemical attack of other hydroxyl groups on the external fullerene surface leading to a much higher hydroxylated system [20]. If one considers now the $\mathrm{C}_{60}(\mathrm{OH})_{16}$ isomer [Fig. 1(h)], both the HOMO and LUMO are more symmetrically distributed on the carbon cage. It is well known that pristine $\mathrm{C}_{60}$ possessess a high electron affinity and its low-lying degenerate LUMO states allow an active participation in free-radical and electron-transfer processes [9,37-39]. Also, we have shown that pure $\mathrm{C}_{60}$ can be stabilized in an aqueous solution via the interaction of its LUMO states with the HOMO states localized in lone pairs of the water molecules [40]. Interestingly, analyzing the singleelectron states of the $\mathrm{C}_{60}(\mathrm{OH})_{24}$ displayed in Fig. 2 (bottom, right) we observe that its $\mathrm{HOMO}$ are completely localized in lone pairs of the adsorbed $\mathrm{OH}$ groups, whereas the LUMO are distributed on the carbon cage. This provides striking evidence that the hydroxyl groups on the fullerene surface
TABLE II. Dipole moments (in $D$ ), polarizabilities, anisotropies (in a.u.), and first hyperpolarizabilities (in a.u.) calculated with B3LYP/6-31G $(d, p)$.

\begin{tabular}{lcccc}
\hline \hline$n$ & $\mu$ & $\alpha$ & $\Delta \alpha$ & $\beta_{\text {tot }}$ \\
\hline 0 & 0.000 & 469 & 0 & 0 \\
1 & 1.038 & 486 & 36 & 378 \\
2 & 1.454 & 486 & 52 & 16 \\
3 & 1.067 & 501 & 81 & 224 \\
4 & 0.000 & 501 & 103 & 0 \\
8 & 0.000 & 537 & 117 & 0 \\
10 & 1.732 & 548 & 207 & 364 \\
16 & 0.000 & 556 & 142 & 5 \\
18 & 0.000 & 698 & 217 & 0 \\
24 & 0.000 & 577 & 1 & 0 \\
32 & 0.000 & & & \\
36 & 5.815 & & & \\
\hline \hline
\end{tabular}

dramatically reduce the reactivity of this fullerenol isomer with the external medium. As noticed by Sayes and coworkers [9], $\mathrm{C}_{60}(\mathrm{OH})_{24}$ presents low oxidative damage to the cell membranes (cytotoxicity) up to its limit of solubility. Thus, the high hydroxylation of fullerenes may reduce their ability to provide reactive oxygen species and, consequently, diminish their cytotoxicity.

\section{B. Depolarized light scattering of fullerenols}

Here, we report both elastic (Rayleigh) and inelastic (Raman) light-scattering properties of fullerenols. Also, as the production of water-soluble forms of these molecules is largely required for bioapplications, we have analyzed their dipole moments, mean dipole polarizabilities, anisotropies, and the first hyperpolarizabilities. Our results are presented in Table II. As expected, the fullerenols exhibit higher polarizabilities than the bare $\mathrm{C}_{60}$ molecule and their values increase slightly with the number of hydroxyl groups attached on the fullerene surface. Of course, considering the 6-31G $(d, p)$ basis set our calculated static polarizability of $\mathrm{C}_{60}$ is 469 a.u., which is around $9 \%$ lower than the experimental value of 517 a.u. [29,41]. Indeed, it is possible to improve considerably this theoretical value by including diffuse functions. For example, Yan and co-workers have recently combined augmented correlation consistent basis sets, such as aug-cc-pVDZ, with the B3LYP functional to obtain a polarizability of 552 a.u. [28] for $\mathrm{C}_{60}$. However, as we are aiming at larger systems, such as $\mathrm{C}_{60}(\mathrm{OH})_{24}$, a higher level of calculation is beyond the applicability of our systematic methodology. Thus, we have calculated all these properties at the B3LYP/6-31G $(d, p)$ level of theory for $\mathrm{C}_{60}(\mathrm{OH})_{n}$ up to $n=24$.

Comparing to the pure $\mathrm{C}_{60}$ molecule, the adsorption of one $\mathrm{OH}$ group leads to a small increase in polarizability, about 17 a.u. Also, the polarizability does not vary when the second $\mathrm{OH}$ group is adsorbed on the fullerene surface. Similarly, for $\mathrm{C}_{60}(\mathrm{OH})_{3}$ and $\mathrm{C}_{60}(\mathrm{OH})_{4}$, the polarizabilities present 
TABLE III. Rayleigh depolarization ratios related to natural, plane-polarized, and circularly polarized incident light and activities (in a.u.) calculated with $\mathrm{B} 3 \mathrm{LYP} / 6-31 \mathrm{G}(d, p)$.

\begin{tabular}{lcccccc}
\hline \hline & $\begin{array}{c}\sigma_{n} \\
\left(\times 10^{-2}\right)\end{array}$ & $\begin{array}{c}\sigma_{p} \\
\left(\times 10^{-2}\right)\end{array}$ & $\begin{array}{c}\sigma_{c} \\
\left(\times 10^{-2}\right)\end{array}$ & $\begin{array}{c}R_{n} \\
\left(\times 10^{7}\right)\end{array}$ & $\begin{array}{c}R_{p \perp} \\
\left(\times 10^{7}\right)\end{array}$ & $\begin{array}{c}R_{p_{\|}} \\
\left(\times 10^{4}\right)\end{array}$ \\
\hline 0 & 0.000 & 0.000 & 0.000 & 0.98 & 0.98 & 0.00 \\
1 & 0.074 & 0.037 & 0.074 & 1.06 & 1.06 & 0.78 \\
2 & 0.152 & 0.076 & 0.153 & 1.07 & 1.06 & 1.62 \\
3 & 0.349 & 0.175 & 0.350 & 1.14 & 1.13 & 3.95 \\
4 & 0.557 & 0.279 & 0.560 & 1.14 & 1.14 & 6.34 \\
8 & 0.630 & 0.316 & 0.634 & 1.31 & 1.31 & 8.23 \\
10 & 1.861 & 0.939 & 1.896 & 1.41 & 1.38 & 25.70 \\
16 & 0.866 & 0.435 & 0.874 & 1.42 & 1.41 & 12.17 \\
18 & 1.263 & 0.636 & 1.280 & 2.26 & 2.23 & 28.15 \\
24 & 0.000 & 0.000 & 0.000 & 1.50 & 1.50 & 0.00 \\
\hline \hline
\end{tabular}

the same values, which are increased by 32 a.u. with respect to $\mathrm{C}_{60}$. From this point, we observe polarizability increases of 68 a.u. in $\mathrm{C}_{60}(\mathrm{OH})_{8}$ and 229 a.u. in $\mathrm{C}_{60}(\mathrm{OH})_{18}$. Interestingly, this latter structure gives a polarizability larger than the $\mathrm{C}_{60}(\mathrm{OH})_{24}$ isomer that possesses a more homogenous distribution of hydroxyl groups on its surface, as indicated by its negligible anisotropy. Among our chosen set of fullerenols, only the $\mathrm{C}_{60}(\mathrm{OH})_{1-3}, \mathrm{C}_{60}(\mathrm{OH})_{10}$, and $\mathrm{C}_{60}(\mathrm{OH})_{36}$ isomers have permanent dipole moments, although the rotation of the hydroxyl groups can easily lead to polar conformations. For instance, in this series the $\mathrm{C}_{60}(\mathrm{OH})_{36}$ isomer gives the largest dipole moment value of $5.815 \mathrm{D}$. This seems to be in accordance with the experimental observation that highly hydroxylated systems have good solubility in an aqueous solution [13]. Due to the interest of these complexes in photonics $[5,8]$, we also report their total first hyperpolarizabilities in Table II. As expected for the totally symmetrical structures $[31,42]$ the total hyperpolarizability vanishes, whereas it presents the highest values for the less symmetrical isomers: $\mathrm{C}_{60} \mathrm{OH}$ (378 a.u.), $\mathrm{C}_{60}(\mathrm{OH})_{3}$ (224 a.u.), and $\mathrm{C}_{60}(\mathrm{OH})_{10}$ (364 a.u.).

In Table III we report the depolarization ratios and activities for Rayleigh scattering. As discussed in Sec. II, these quantities are connected with the squares of the mean dipole polarizability and anisotropy of the molecular system [23]. Thus, changes in these properties lead to appreciable variations in the depolarization ratios of the fullerenols. We have computed all the components of the depolarization due to natural, plane-polarized, and circularly polarized incident light on the systems shown in Figs. 1(a)-1(j). As normally expected for the more anisotropic cases we have obtained the largest depolarization ratios, although in the case of $\mathrm{C}_{60}(\mathrm{OH})_{18}$ the depolarization ratio is about $32 \%$ smaller than in $\mathrm{C}_{60}(\mathrm{OH})_{10}$. Interestingly, this latter isomer also presents the highest values for all the components of depolarization in this fullerenol series. Notice that Rayleigh activity associated with natural $\left(R_{n}\right)$ and perpendicularly polarized $\left(R_{p \perp}\right)$ light varies slightly with the $\mathrm{OH}$ groups number, whereas the activity related to the polarized light $\left(R_{p \|}\right)$ in the same plane of scattering increases with the anisotropy, giving appreciably high values for $\mathrm{C}_{60}(\mathrm{OH})_{10}$ and $\mathrm{C}_{60}(\mathrm{OH})_{18}$.
TABLE IV. Raman intensities (in a.u.) and depolarization ratios related to plane-polarized and natural incident light calculated with B3LYP/ 6-31G $(d, p)$.

\begin{tabular}{lcccccccc}
\hline \hline & \multicolumn{2}{c}{ C-C stretching modes } & & \multicolumn{3}{c}{ C-O stretching modes } \\
\cline { 7 - 8 }$n$ & $A_{n}$ & $\rho_{p}$ & $\rho_{n}$ & & $A_{n}$ & $\rho_{p}$ & $\rho_{n}$ \\
\hline 0 & 412 & 0.00 & 0.00 & & & \\
1 & 371 & 0.00 & 0.01 & & 33 & 0.32 & 0.48 \\
2 & 433 & 0.00 & 0.00 & & 37 & 0.30 & 0.46 \\
3 & 332 & 0.00 & 0.00 & & 57 & 0.36 & 0.53 \\
4 & 430 & 0.00 & 0.01 & & 74 & 0.31 & 0.47 \\
8 & 652 & 0.07 & 0.12 & & 172 & 0.07 & 0.12 \\
10 & 454 & 0.02 & 0.05 & & 165 & 0.07 & 0.13 \\
16 & 202 & 0.07 & 0.13 & & 187 & 0.05 & 0.10 \\
18 & 950 & 0.41 & 0.59 & & 146 & 0.28 & 0.44 \\
24 & 307 & 0.00 & 0.00 & 21 & 0.54 & 0.70 \\
\hline \hline
\end{tabular}

As a complement of the depolarized light scattering, Raman depolarization ratios and intensities were also calculated at the $\mathrm{B} 3 \mathrm{LYP} / 6-31 \mathrm{G}(d, p)$ level. As well known, these are differential inelastic properties and depend on each vibration normal mode of the analyzed system $[35,36]$. Thus, we present the degree of depolarization of planar and natural incident light ( $\rho_{p}$ and $\rho_{n}$, respectively) for two selected vibrational modes of each fullerenol in Table IV. Following in this section, we will discuss the complete vibrational analysis of the fullerenols with the ir and Raman absorption spectra. Here, we show the calculated Raman depolarization ratios related to the $\mathrm{C}-\mathrm{C}$ (resembling the $A_{g}$ pentagonal pinch mode of $\mathrm{C}_{60}$ [43]) and $\mathrm{C}-\mathrm{O}$ stretching modes of the fullerenols. As can be noticed in Table IV, the relevant depolarization ratios due to the $\mathrm{C}-\mathrm{C}$ stretches appear from $\mathrm{C}_{60}(\mathrm{OH})_{8}$ up to $\mathrm{C}_{60}(\mathrm{OH})_{18}$, whereas the largest depolarization ratios of the $\mathrm{C}-\mathrm{O}$ stretches occur for $\mathrm{C}_{60}(\mathrm{OH})_{1-4}$ and also for $\mathrm{C}_{60}(\mathrm{OH})_{18}$ and $\mathrm{C}_{60}(\mathrm{OH})_{24}$. Now, regarding the $\mathrm{C}-\mathrm{C}$ stretching modes, incident light is more depolarized for $\mathrm{C}_{60}(\mathrm{OH})_{18}$ with the highest Raman intensity. However, considering the $\mathrm{C}-\mathrm{O}$ stretching modes, $\mathrm{C}_{60}(\mathrm{OH})_{24}$ gives the largest depolarization ratios with the lowest Raman intensity in this fullerenol series. At this point, it is important to mention that complementary determinations of other properties are necessary to distinguish between these different complexes. In this sense, all the vibrational modes will be next analyzed within the ir and Raman spectra.

\section{Vibrational spectra of the fullerenols}

In order to make good theoretical predictions of the vibrational spectra of fullerenols, the accuracy of the $\mathrm{B} 3 \mathrm{LYP} / 6-31 \mathrm{G}(d, p)$ method is assessed by calculating the vibrational spectrum of $\mathrm{C}_{60}$ and comparing it with available experimental data [44-46]. For this molecule the 174 vibrational modes can be reduced into the irreducible representations (irrep's) of the $I_{h}$ point group, which gives 46 distinct modes as follows [43]: 
TABLE V. Group-theoretical assignment of the calculated ir and Raman bands (in $\mathrm{cm}^{-1}$ ) of $\mathrm{C}_{60}$ in comparison with different sets of experimental data and early DFT calculations.

\begin{tabular}{lccccccc}
\hline \hline & \multicolumn{2}{c}{ Even parity } & & & & & \\
& & & & & & \\
\end{tabular}

${ }^{\mathrm{a}}$ Reference [47].

${ }^{b}$ References [44] and/or [45].

$\Gamma=2 A_{g}+3 T_{1 g}+4 T_{2 g}+6 G_{g}+8 H_{g}+A_{u}+4 T_{1 u}+5 T_{2 u}+6 G_{u}$ $+7 H_{u}$.

All these calculated fundamental modes are given in Table $\mathrm{V}$, along with the experimental frequencies and group theory analysis. Additionally, our calculated vibrational spectrum of $\mathrm{C}_{60}$ is compared to early results [47] obtained with allelectron local-density-functional methods. As seen in Table $\mathrm{V}$, these DFT calculations give excellent agreement with the experimental data available for Raman and infrared [44-47] spectra. For instance, the maximum deviation of our calculated active frequencies $(3 \%)$ corresponds to the $H_{g}(8)$ mode of $1618 \mathrm{~cm}^{-1}$, as compared to the experimental value of $1570 \mathrm{~cm}^{-1}$ [44]. However, as reported in Ref. [44] the experimental measurement of this mode is liable to different resonance conditions. Our calculated ir and Raman absorption spectra are shown in Fig. 4. In the ir spectrum we obtain peaks at $539,588,1212$, and $1461 \mathrm{~cm}^{-1}$. As is well known, these values are only the ir active modes belonging to the $T_{1 u}$ symmetry of the $I_{h}$ point group of the $\mathrm{C}_{60}$ molecule. These results are in line with experimental ir spectroscopic data [45], exhibiting peaks located at 526, 576, 1182, and $1428 \mathrm{~cm}^{-1}$.

As can be seen from the above results, the present level of theory leads to a good result for the calculated ir spectrum of fullerene compared to the use of smaller basis sets (e.g., 6-21 $\mathrm{G}$ giving 540 CGFs for $\mathrm{C}_{60}$ [20]). In our case we utilize 900 CGFs for $\mathrm{C}_{60}$ through the $6-31 \mathrm{G}(d, p)$ basis set. Thus, we also expect to obtain good estimates for the calculated Raman spectra. In this case, the bare $\mathrm{C}_{60}$ molecule presents 10 fundamental Raman active modes, two of them belonging to the $A_{g}$ symmetry and 8 belonging to the $H_{g}$ symmetry of the $I_{h}$ point group. As indicated in Fig. 4 (bottom), the calculated band intensities corresponding to the $H_{g}$ modes at 438, 720, 787,1125 , and $1455 \mathrm{~cm}^{-1}$ are weaker than the absorption peaks of the other modes. This indicates that the $H_{g}$ modes will be weakly coupled to the change in the electronic distribution of the fullerene surface [44]. Once again our calcu- 


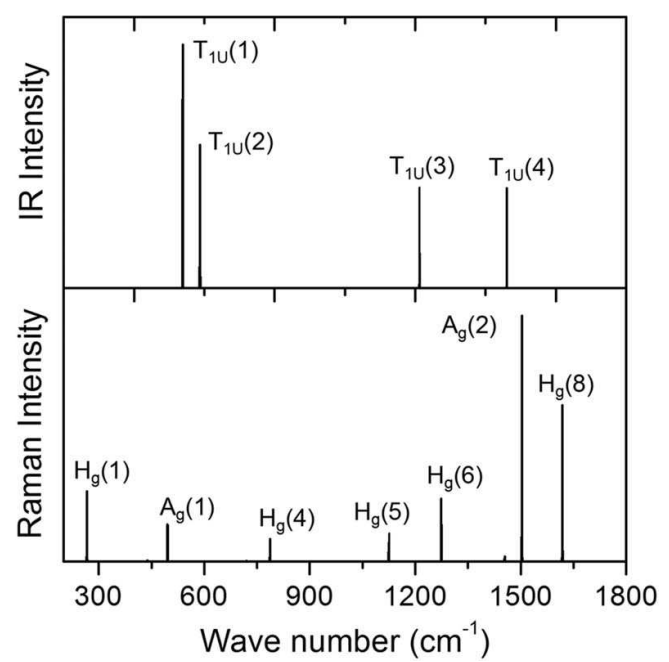

FIG. 4. (Top) ir spectrum and (bottom) Raman spectrum of the pristine $\mathrm{C}_{60}$ molecule calculated at the B3LYP/6-31G $(d-p)$ level of theory. The intensities of the Raman active modes $H_{g}(2), H_{g}(3)$, and $H_{g}(7)$ are too weak to be represented in the spectrum given above.

lated values are in excellent agreement with Raman spectroscopic experiments as given in Table V.

Now, the adsorption of one hydroxyl group on the fullerene surface provokes a lowering of symmetry of the carbon cage [see Figs. 1(a) and 1(b) for comparison]. For example, the lengths of the nearest-neighbor $\mathrm{C}-\mathrm{C}$ bonds to the $\mathrm{OH}$-adsorbed site are increased by approximately $0.1 \AA$ compared to the ones around the nonfunctionalized carbon atoms. The optimized $\mathrm{C}-\mathrm{O}$ and $\mathrm{O}-\mathrm{H}$ bond lengths are, respectively, 1.431 and $0.969 \AA$, along with a $\mathrm{C}-\mathrm{O}-\mathrm{H}$ angle of $107.4^{\circ}$ in the $\mathrm{C}_{60} \mathrm{OH}$ molecule. This geometric deformation leads to a more complex distribution of vibrational modes in the fullerenol, displayed in the ir and Raman spectra of Fig. 5. As shown in the calculated ir spectrum (top), the significant peak at $1045 \mathrm{~cm}^{-1}$ corresponds to the $\mathrm{C}-\mathrm{O}$ stretching mode, whereas the weaker ir absorption at $3780 \mathrm{~cm}^{-1}$ corresponds to the $\mathrm{O}-\mathrm{H}$ stretching mode of $\mathrm{C}_{60} \mathrm{OH}$. In the Raman spectrum (bottom) the intensities of these modes are opposite. There is still a contribution of various stretching and bending modes around the $\mathrm{C}-\mathrm{O}$ stretch absorption, resulting from the nearest-neighbor carbon atoms to the functionalized site. Interestingly, we observe that the characteristic Raman absorption at $1495 \mathrm{~cm}^{-1}$, resembling the $A_{g}$ pentagonal pinch mode of pure $\mathrm{C}_{60}$, is slightly redshifted by $8 \mathrm{~cm}^{-1}$ upon single $\mathrm{OH}$ adsorption. Also, the fivefold degenerate Raman active absorptions $\left(H_{g}\right)$ at $1618 \mathrm{~cm}^{-1}$ are split in $\mathrm{C}_{60} \mathrm{OH}$ into the range of $1607-1614 \mathrm{~cm}^{-1}$, as indicated in Fig. 5 (bottom). Note that a similar pattern is observed in the low wave number absorptions $\left(H_{g}\right)$ at $266 \mathrm{~cm}^{-1}$ of $\mathrm{C}_{60}$. In this case, the effect of the adsorbed $\mathrm{OH}$ group on the fullerene surface is to split this mode in distinct frequencies at 251, 253, 257, and $266 \mathrm{~cm}^{-1}$ (the latter being twofold degenerate). We also observe an intense absorption at $296 \mathrm{~cm}^{-1}$, due to the $\mathrm{OH}$ torsion mode around the $\mathrm{C}-\mathrm{O}$ bond, in the ir spectrum of $\mathrm{C}_{60} \mathrm{OH}$.

In general, it is very difficult to assign the different vibrational modes of fullerenols. The situation becomes more

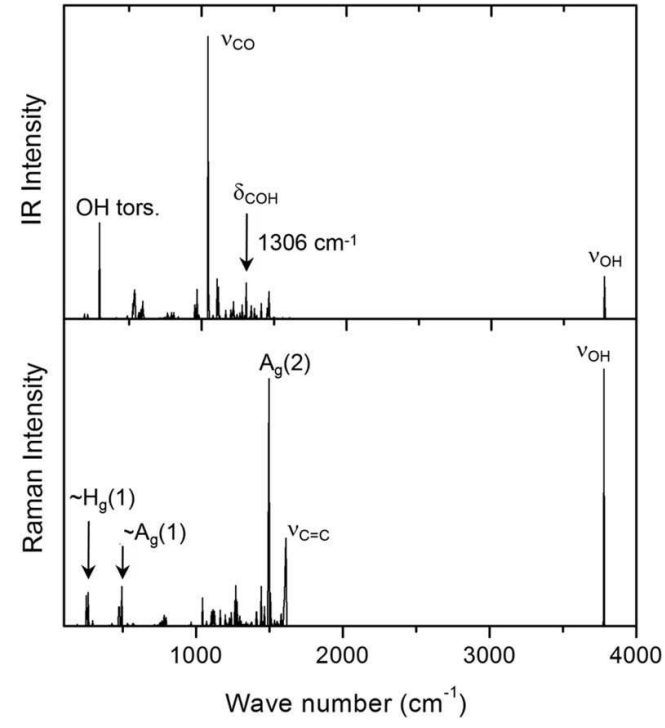

FIG. 5. (Top) ir spectrum and (bottom) Raman spectrum of the $\mathrm{C}_{60} \mathrm{OH}$ molecule calculated at the B3LYP/6-31G $(d, p)$ level of theory. Peaks marked with $\nu$ and $\delta$ refer to the typical stretching and bending modes, respectively, as given on the panels.

complicated in higher hydroxylated systems [13,14]. However, the calculated ir and Raman characteristic absorptions can aid one in understanding the structural features of these molecules. Then, we present some theoretical spectra at the same level of DFT described above for $\mathrm{C}_{60}(\mathrm{OH})_{n}$ systems with $n=2,4,8,10,16$, and 24 in Fig. 6. For clarity, we show six representative ir spectra of the fullerenols in Figs. 6(a)-6(f) (left). We observe the characteristic $\mathrm{C}-\mathrm{O}$ stretching absorption $\left(1063-1097 \mathrm{~cm}^{-1}\right)$ in all these molecules. At this point, we must stress that the calculated frequencies are in good accordance with the locations of the assigned to $\mathrm{C}-\mathrm{O}$ absorption band in the experimental ir spectra of $\mathrm{C}_{60}(\mathrm{OH})_{18-20}\left(\right.$ at $\left.1055 \mathrm{~cm}^{-1}\right)$ [14], $\mathrm{C}_{60}(\mathrm{OH})_{24-28}$ fullerenols $\left(1070-1090 \mathrm{~cm}^{-1}\right)$ [45], as well as with the $\mathrm{C}-\mathrm{O}$ absorption (at about $1088 \mathrm{~cm}^{-1}$ ) in higher polyhydroxylated fullerenes [13].

The calculated $\mathrm{O}-\mathrm{H}$ stretching absorptions for our fullerenol series are in the range of $3760-3792 \mathrm{~cm}^{-1}$. Experimentally, this band has been observed as an intense broad absorption band at about $3430 \mathrm{~cm}^{-1}$ in $\mathrm{C}_{60}(\mathrm{OH})_{24-28} \mathrm{com}-$ pounds [45] or at about $3410 \mathrm{~cm}^{-1}$ in highly hydroxylated fullerenes [13]. Normally, it is much more difficult to describe theoretically the $\mathrm{O}-\mathrm{H}$ vibrational stretches without using a scaling factor. However, an important feature must be noticed here with respect to this absorption in the ir spectrum of $\mathrm{C}_{60}(\mathrm{OH})_{10}$. For this structure [Fig. 1(g)], which exhibits five alternate hydrogen bonds $(1.898 \AA)$, we observe two distinct absorptions: one at $3657 \mathrm{~cm}^{-1}$, corresponding to the $\mathrm{O}-\mathrm{H}$ hydrogen bonded stretch, and the other at $3805 \mathrm{~cm}^{-1}$, corresponding to the free $\mathrm{O}-\mathrm{H}$ stretch, as shown in Fig. 6(d) (left). Thus, it is expected that the coupling between adjacent $\mathrm{OH}$ groups in highly hydroxylated systems will yield stretch modes in a lower wave number region. This effect should be augmented with the increase of the number of hydrogen bonds on the fullerene surface. 

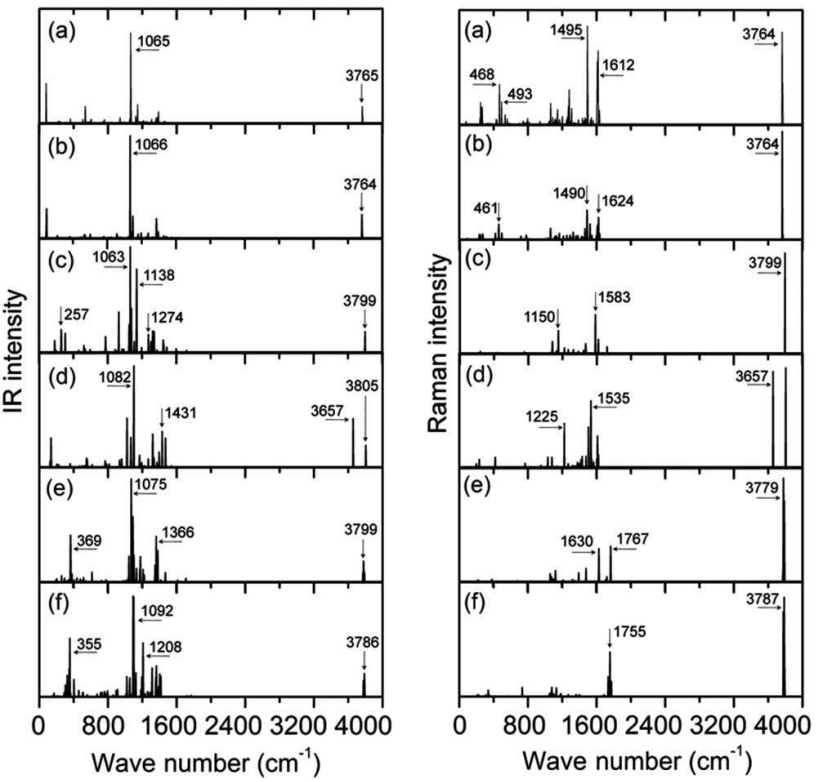

FIG. 6. (Left) ir and (right) Raman spectra calculated for different fullerenols at the $\mathrm{B} 3 \mathrm{LYP} / 6-31 \mathrm{G}(d, p)$ level of theory. (a) $\mathrm{C}_{60}(\mathrm{OH})_{2}, \quad$ (b) $\mathrm{C}_{60}(\mathrm{OH})_{4}, \quad$ (c) $\mathrm{C}_{60}(\mathrm{OH})_{8}, \quad$ (d) $\mathrm{C}_{60}(\mathrm{OH})_{10}, \quad$ (e) $\mathrm{C}_{60}(\mathrm{OH})_{16}$, and (f) $\mathrm{C}_{60}(\mathrm{OH})_{24}$. The typical ir and Raman absorptions in fullerenols are indicated on the panels. These are the stretching modes of the $\mathrm{OH}$ groups $\left(3760-3792 \mathrm{~cm}^{-1}\right)$; the ir-active $\mathrm{C}-\mathrm{O}$ stretching modes $\left(1063-1097 \mathrm{~cm}^{-1}\right)$; the ir-active $\mathrm{C}-\mathrm{O}-\mathrm{H}$ bending mode at $1138,1274,1431,1366$, and $1208 \mathrm{~cm}^{-1}$; the ir-active $\mathrm{OH}$ torsions at 257,369 , and $355 \mathrm{~cm}^{-1}$; the Raman-active breath modes at 468,493 , and $461 \mathrm{~cm}^{-1}$; and the Raman-active $\mathrm{C}=\mathrm{C}$ stretching modes $\left(1150-1755 \mathrm{~cm}^{-1}\right)$.

More important information obtained from the calculated ir spectra of $\mathrm{C}_{60}(\mathrm{OH})_{n}$ is the absorption relative to the $\mathrm{C}-\mathrm{O}-\mathrm{H}$ bending modes. For example, in Fig. 6(c) (left) for $n=8$ we observe an intense ir active absorption at $1138 \mathrm{~cm}^{-1}$ along with a less intense absorption at $1303 \mathrm{~cm}^{-1}$, corresponding to the combined $(\mathrm{OH})_{4}-(\mathrm{OH})_{4}$ asymmetric bending modes, and a less intense absorption at $1274 \mathrm{~cm}^{-1}$, corresponding to the independent asymmetric bending modes of $4 \mathrm{OH}$ groups at each pole of $\mathrm{C}_{60}(\mathrm{OH})_{8}$. As reported in Ref. [16], these types of vibration give rise to strong ir absorptions in highly hydroxylated fullerenes at about $1412 \mathrm{~cm}^{-1}$. Indeed, this becomes more explicit in the calculated ir spectrum of $\mathrm{C}_{60}(\mathrm{OH})_{24}$ [see Fig. 6(f), left], which shows different combinations of the $\mathrm{C}-\mathrm{O}-\mathrm{H}$ bending modes with more intense peak at $1388 \mathrm{~cm}^{-1}$, and twofold moderate absorptions at 1403 and $1417 \mathrm{~cm}^{-1}$. From the theoretical ir spectra of higher hydroxylated fullerenes, we also observe relatively strong absorptions located at low frequencies. This is the case of $\mathrm{C}_{60}(\mathrm{OH})_{16}$ and $\mathrm{C}_{60}(\mathrm{OH})_{24}$ [Figs. 6(e) and 6(f)] exhibiting absorptions at 369 and $355 \mathrm{~cm}^{-1}$, respectively, corresponding to the combinations of $\mathrm{O}-\mathrm{H}$ torsions.

To give a more detailed description of the vibrational spectra of these molecules, we also report our calculated Raman spectra for the $\mathrm{C}_{60}(\mathrm{OH})_{n}$ systems with $n=2,4,8,10,16$ and 24 in Fig. 6 (right). Despite the extensive research in- volving different fullerenols, no systematic Raman spectroscopy studies on these molecules seems to be reported to date [25]. In this sense, the Raman spectra are displayed in Figs. 6(a)-6(f) (right). Now, the $\mathrm{O}-\mathrm{H}$ stretching modes present very strong absorptions, as can be noted in the spectra of the compounds. In the Raman spectrum of $\mathrm{C}_{60}(\mathrm{OH})_{2}$ [Fig. 6(a), right] the residue of the $H_{g}(1)$ vibrational modes still remain as five distinct absorptions at 244, 249, 255, and $267 \mathrm{~cm}^{-1}$. However, these tend to be reduced with the increase of hydroxylation. A similar effect is observed in the breath modes at 468 and $493 \mathrm{~cm}^{-1}$ of $\mathrm{C}_{60}(\mathrm{OH})_{2}$, as compared to the higher hydroxylated fullerenes. The main features of the Raman spectra of the fullerenol molecules with $n=2-10$ can be observed at around 1500 and $1620 \mathrm{~cm}^{-1}$, corresponding, respectively, to the $A_{g}(2)$ and $H_{g}(8)$ modes in bare $\mathrm{C}_{60}$. For example, a very intense absorption at $1497 \mathrm{~cm}^{-1}$ is observed in $\mathrm{C}_{60}(\mathrm{OH})_{2}$. Also, these modes present different combinations in $\mathrm{C}_{60}(\mathrm{OH})_{4}$ (at 1490 and $\left.1624 \mathrm{~cm}^{-1}\right), \mathrm{C}_{60}(\mathrm{OH})_{8}$ (at 1471 and $1484 \mathrm{~cm}^{-1}$ ), and $\mathrm{C}_{60}(\mathrm{OH})_{10}$ (at 1509, 1535, and $1610 \mathrm{~cm}^{-1}$ ). On the other hand, these bands are not observed in higher hydroxylated systems, which present more localized absorptions at 1630 and $1767 \mathrm{~cm}^{-1}$ in $\mathrm{C}_{60}(\mathrm{OH})_{16}$ and at 1736 and $1755 \mathrm{~cm}^{-1}$ in $\mathrm{C}_{60}(\mathrm{OH})_{24}$. These absorptions correspond to the $\mathrm{C}=\mathrm{C}$ stretching modes between nonfunctionalized carbon sites.

\section{SUMMARY}

In this work we have employed an appropriate DFT level capable of describing diverse molecular properties of fullerenols containing more than 30 hydroxyl groups distributed on the fullerene surface. A systematic study has been carried out on completely optimized structures at the B3LYP/ $6-31 \mathrm{G}(d, p)$ level of theory. This method has been chosen as a compromise between accuracy and capability to treat highly hydroxylated fullerenes, such as $\mathrm{C}_{60}(\mathrm{OH})_{36}$. The calculated depolarization ratios of the scattered light suggest that such studies may be especially valuable in the detailed analysis of structure and behavior of these molecules. The vibrational spectra were calculated and hence the ir and Raman absorption spectra were obtained, giving a good correspondence with the expected changes in the spectral absorptions of highly hydroxylated fullerenes. We have analyzed a wide range of wave number in order to assess the effects of hydroxyl groups on the vibrational properties of fullerenols, comparing them to the pure fullerene spectra. As shown, the spectral patterns closely resemble each other, albeit the relative intensities differ rather, suggesting a difference in the number of adsorbed hydroxyl groups. Indeed, all these findings indicate that the present theoretical calculations may be successfully used to elucidate the main structural features of synthesized fullerenols.

\section{ACKNOWLEDGMENTS}

This work has been partially supported by the Brazilian agencies FAPESB (Bahia), FAPESP (São Paulo), and CNPq. 
[1] V. Jacevic, V. Djordjevic-Milic, V. Dragojevic-Simic, N. Radic, B. Govedarica, S. Dobric, B. Srdjenovic, R. Injac, A. Djordjevic, and V. Vasovic, Toxicol. Lett. 172, S146 (2007).

[2] S. Trajkovic, S. Dobric, V. Jacevic, V. Dragojevic-Simic, Z. Milovanovic, and A. Dordevic, Colloids Surf., B 58, 39 (2007).

[3] M. P. Gelderman-Fuhrmann, O. Simakova, S. F. Siddiqui, A. C. Vostal, and J. Simak, Blood 108, 511A (2006).

[4] A. R. Badireddy, E. M. Hotze, S. Chellam, P. Alvarez, and M. R. Wiesner, Environ. Sci. Technol. 41, 6627 (2007).

[5] M. E. Rincon, R. A. Guirado-Lopez, J. G. Rodriguez-Zavala, and M. C. Arenas-Arrocena, Sol. Energy Mater. Sol. Cells 87, 33 (2005).

[6] B. Vileno, P. R. Marcoux, M. Lekka, A. Sienkiewicz, T. Feher, and L. Forro, Adv. Funct. Mater. 16, 120 (2006).

[7] S. Aikawa, Y. Yoshida, S. Nishiyama, H. Noguchi, and A. Shoji, Mol. Cryst. Liq. Cryst. 445, 315 (2006).

[8] R. A. Guirado-Lopez and M. E. Rincon, J. Chem. Phys. 125, 154312 (2006)

[9] C. M. Sayes, J. D. Fortner, W. Guo, D. Lyon, A. M. Boyd, K. D. Ausman, Y. J. Tao, B. Sitharaman, L. J. Wilson, J. B. Hughes, J. L. West, and V. L. Colvin, Nano Lett. 4, 1881 (2004).

[10] K. D. Pickering and M. R. Wiesner, Environ. Sci. Technol. 39, 1359 (2005).

[11] G. Bogdanovic, V. Kojic, A. Dordevic, J. Canadanovic-Brunet, M. Vojinovic-Miloradov, and V. V. Baltic, Toxicol. In Vitro 18, 629 (2004).

[12] A. Isakovic, Z. Markovic, B. Todorovic-Markovic, N. Nikolic, S. Vranjes-Djuric, M. Mirkovic, M. Dramicanin, L. Harhaji, N. Raicevic, Z. Nikolic, and V. Trajkovic, Toxicol. Sci. 91, 173 (2006).

[13] K. Kokubo, K. Matsubayashi, H. Tategaki, H. Takada, and T. Oshima, ACS Nano 2, 327 (2008).

[14] G. C. Alves, L. O. Ladeira, A. Righi, K. Krambrock, H. D. Calado, R. P. F. Gil, and M. V. B. Pinheiro, J. Braz. Chem. Soc. 17, 1186 (2006)

[15] E. E. Fileti, R. Rivelino, F. B. Mota, and T. Malaspina, Nanotechnology 19, 365703 (2008).

[16] G. M. Xing, J. Zhang, Y. L. Zhao, J. Tang, B. Zhang, X. F. Gao, H. Yuan, L. Qu, W. B. Cao, Z. F. Chai, K. Ibrahim, and R. Su, J. Phys. Chem. B 108, 11473 (2004).

[17] In practice very efficient searching procedures would be required to scan the whole potential energy surface. These methods are usually based on molecular dynamics (MD); however, this depends on the existence of reliable empirical potentials. Then, $a b$ initio calculations could be used to verify the quality of the force fields. Of course, the combination of inaccurate empirical potentials with MD simulations will lead to inaccurate structures as well.

[18] B.-C. Wang and C.-Y. Cheng, J. Mol. Struct.: THEOCHEM 391, 179 (1997).

[19] Z. Slanina, X. Zhao, L. Y. Chiang, and E. Osawa, Int. J. Quantum Chem. 74, 343 (1999).

[20] J. G. Rodriguez-Zavala and R. A. Guirado-Lopez, Phys. Rev. B 69, 075411 (2004).

[21] J. G. Rodriguez-Zavala and R. A. Guirado-Lopez, J. Phys. Chem. A 110, 9459 (2006).

[22] P. Karamanis, C. Pouchan, and G. Maroulis, Phys. Rev. A 77, 013201 (2008).
[23] E. E. Fileti, R. Rivelino, and S. Canuto, J. Phys. B 36, 399 (2003).

[24] E. E. Fileti, M. A. Castro, and S. Canuto, Chem. Phys. Lett. 452, 54 (2008).

[25] P. Scharff, C. Siegmund, K. Risch, I. Lysko, O. Lysko, A. Zherebetskyy, A. Ivanisik, A. Gorchinskiy, and E. Buzaneva, Fullerenes, Nanotubes, Carbon Nanostruct. 13, 497 (2005).

[26] A. D. Becke, J. Chem. Phys. 98, 5648 (1993).

[27] C. Lee, W. Yang, and R. G. Parr, Phys. Rev. B 37, 785 (1988).

[28] H. Yan, S. Yu, X. Wang, Y. He, W. Huang, and M. Yang, Chem. Phys. Lett. 456, 223 (2008).

[29] F. Rabilloud, R. Antoine, M. Broyer, I. Compagnon, P. Dugourd, D. Rayane, F. Calvo, and F. Spiegelman, J. Phys. Chem. C 111, 17795 (2007).

[30] M. J. Frisch, G. W. Trucks, H. B. Schlegel, G. E. Scuseria, M. A. Robb, J. R. Cheeseman, J. A. Montgomery, Jr., T. Vreven, K. N. Kudin, J. C. Burant, J. M. Millam, S. S. Iyengar, J. Tomasi, V. Barone, B. Mennucci, M. Cossi, G. Scalmani, N. Rega, G. A. Petersson, H. Nakatsuji, M. Hada, M. Ehara, K. Toyota, R. Fukuda, J. Hasegawa, M. Ishida, T. Nakajima, Y. Honda, O. Kitao, H. Nakai, M. Klene, X. Li, J. E. Knox, H. P. Hratchian, J. B. Cross, V. Bakken, C. Adamo, J. Jaramillo, R. Gomperts, R. E. Stratmann, O. Yazyev, A. J. Austin, R. Cammi, C. Pomelli, J. W. Ochterski, P. Y. Ayala, K. Morokuma, G. A. Voth, P. Salvador, J. J. Dannenberg, V. G. Zakrzewski, S. Dapprich, A. D. Daniels, M. C. Strain, O. Farkas, D. K. Malick, A. D. Rabuck, K. Raghavachari, J. B. Foresman, J. V. Ortiz, Q. Cui, A. G. Baboul, S. Clifford, J. Cioslowski, B. B. Stefanov, G. Liu, A. Liashenko, P. Piskorz, I. Komaromi, R. L. Martin, D. J. Fox, T. Keith, M. A. Al-Laham, C. Y. Peng, A. Nanayakkara, M. Challacombe, P. M. W. Gill, B. Johnson, W. Chen, M. W. Wong, C. Gonzalez, and J. A. Pople, Gaussian, Inc., Wallingford CT, 2004, GAUSSIAN 03, Revision D.01 (Gaussian, Inc., Wallingford, CT, 2004).

[31] A. D. Buckingham, Adv. Chem. Phys. 12, 107 (1967).

[32] G. H. F. Diercksen and A. J. Sadlej, Chem. Phys. Lett. 153, 93 (1988).

[33] G. Herzberg, Infrared and Raman Spectra of Polyatomic molecules (van Nostrand, Princeton, NJ, 1945).

[34] As usual, Rayleigh intensity associated with the planepolarized light, $R_{p}$, has been split into two orthogonal parts: $R_{p \perp}$, which corresponds to the perpendicularly polarized light into the scattering plane; and $R_{p \|}$, which corresponds to the polarized light in the same plane of scattering.

[35] H. Suzuki, Prog. Theor. Phys. 62, 936 (1979).

[36] H. A. Szymanski, Raman Spectroscopy (Plenum Press, New York, 1967).

[37] H. Mohan, D. K. Palit, J. P. Mittal, L. Y. Chiang, K.-D. Asmus, and D. M. Guldi, J. Chem. Soc., Faraday Trans. 94, 359 (1998).

[38] D. M. Guldi, H. Hungerbuhler, and K.-D. Asmus, J. Phys. Chem. B 103, 1444 (1999).

[39] I. Nakanishi, S. Fukuzumi, T. Konishi, K. Ohkubo, M. Fujitsuka, O. Ito, and N. Miyata, J. Phys. Chem. B 106, 2372 (2002).

[40] R. Rivelino and F. B. Mota, Nano Lett. 7, 1526 (2007).

[41] R. Antoine, P. Dugourd, D. Rayane, E. Benichou, M. Broyer, F. Chandezon, and C. Guet, J. Chem. Phys. 110, 9771 (1999).

[42] G. Maroulis, Chem. Phys. Lett. 444, 44 (2007).

[43] M. S. Dresselhaus, G. Dresselhaus, and P. Eklund, in Science 
of Fullerene and Carbon Nanotubes (Academic Press, Sandiego, CA, 1996).

[44] S. H. Gallager, R. S. Armstrong, W. A. Clucas, P. A. Lay, and C. A. Reed, J. Phys. Chem. A 101, 2960 (1997).

[45] M. E. Rincon, H. Hu, J. Campos, and J. Ruiz-Garcia, J. Phys.
Chem. B 107, 4111 (2003).

[46] Z. Niu and Y. Fang, Vib. Spectrosc. 43, 415 (2007).

[47] X. Q. Wang, C. Z. Wang, and K. M. Ho, Phys. Rev. B 48, 1884 (1993). 